\title{
Construction of Optimal Odd Fractional Designs in Mixed Series Experiment Using Semi Latin Square
}

\author{
Williams, E. E. (Corresponding author) \\ Department of Maths, Stats and Computer Science, University of Calabar, Calabar, Nigeria \\ P.M.B. 1115, Calabar, Nigeria \\ Tel: 234-803-547-0457 E-mail: edemwilliam@yahoo.com
}

Akpan, S. S.

Department of Maths, Stats and Computer Science, University of Calabar, Calabar, Nigeria

P.M.B. 1115, Calabar, Nigeria.

Tel: $234-803-380-8695$

Ugbe, T. A.

Department of Maths, Stats and Computer Science

University of Calabar, Calabar, Nigeria

Nduka, E. C.

Department of Mathematics \& Statistics, University of Port Harcourt

Port Harcourt, Nigeria

\begin{abstract}
A new technique for the construction of optimal odd fractional designs in mixed series experiment is developed. The construction begins by classifying all the experimental points in the experimental region into groups where the levels of each factor is centred at zero, thereafter the $\mathrm{P}$ point trial designs is formed by picking the support points from the first group to the last depending on the size of the designs using some optimality checks. The technique obtains a P-point optimal design using loss of information as an optimality Criteria. Numerical illustrations confirm the effectiveness of this technique.
\end{abstract}

Keywords: Latin Square, Mixed Experiment, Optimality Criteria, Optimality Check, Odd-Fractional Designs

\section{Introduction}

There are many experiments designed to study the effects of variables largely under the control of the experimenter on other variables which are functions of the controlled variables (Raghavarao, 1971). The controlled variables are called factors and the functions are the response variables. The experiment is conducted by changing the settings of the factors and observing the changes which result in the response variables. The settings of a factor for which observations are made on the response variables are called the levels of the factors. (Pazman, 1987)

A complete factorial experimental design requires that observations be made on the response variables for all combinations of the levels of the factors. These combinations will be referred to as treatment combinations. A fractional factorial experimental design requires that only a fraction of these treatment combinations be studied. (Meyer, 1971)

Catalogs of fractional factorial designs for the case when all factors have two levels, and three levels are mostly not available. In symmetrical factorial experiment, we can confound higher order interactions without losing any information on main effects. In mixed factorials, pm x qn we can confound $\mathrm{r}$ independent effects and interactions of factors at $\mathrm{p}$ levels and $s$ independent effects and interactions of factors at q levels in blocks of size pm-rqn-s. White and Hulquist (1995) has given methods for the designs and analysis of confounding plans for the pm $\mathrm{x}$ qn factorial experiment, $\mathrm{p}$ and $\mathrm{q}$ primes, using a technique of combining elements from distinct finite fields. An equivalent theoretical base utilizing ideal theory was obtained by Raktae (1999) and Banjere (1970) showed that it will be possible to provide the confounding plans for mixed factorials of the types $5 \mathrm{n} \times 8 \mathrm{~m}$, where $\mathrm{n}$ and $\mathrm{m}$ are any two positive integers. The aim of this paper is to present a methodology which will yield optimal odd fractional designs in mixed series experiment using semi-Latin squares. 


\section{Methodology}

For a mixed series experiment, an experimental treatment table is displayed to show the different factor levels in the experiment, most often pseudo factors are used to complete the table plans while the incomplete Latin square plan can be superimposed for the group formation. This criterion function shows how much information can be accounted for a particular treatment effect while the use of the optimality checks gives some guide on the choice of designs that are close to optimality in a class of admissible designs. (John, 1980)

Consider a 3 x 5 experiment; the treatment plan is as displayed in table 1. From table 1 the corresponding Latin square plan is shown below, where the dimension is in accordance with higher level of one of the factors involved.

$\begin{array}{lllll}a & b & c & d & e \\ b & c & d & e & a \\ c & d & e & a & b \\ e & a & b & c & d\end{array}$

By superimposing this Latin square plan on the experimental treatment table, the groups are formed by picking a complete directrix of the alphabetical arrangement of the Latin square plan. This is shown below.

The resulting groups are given below.

$$
\left[\begin{array}{cc}
-1 & -2 \\
y & 1 \\
0 & -1 \\
z & 2 \\
1 & 0
\end{array}\right]\left[\begin{array}{cc}
-1 & -1 \\
y & 2 \\
0 & 0 \\
1 & 1 \\
z & -2
\end{array}\right] \quad\left[\begin{array}{cc}
-1 & 0 \\
y & 2 \\
0 & 1 \\
z & -1 \\
1 & 2
\end{array}\right] \quad\left[\begin{array}{cc}
-1 & -1 \\
y & -1 \\
0 & 2 \\
z & 0 \\
1 & -2
\end{array}\right]\left[\begin{array}{cc}
-1 & 2 \\
y & 0 \\
0 & -2 \\
z & 1 \\
1 & -1
\end{array}\right]
$$

From the above groups, the desired P-point treatment designs are formed, where the pseudo-support points are discarded and the real support points are used to make up the P-point desired designs. In the class of admissible designs formed, the optimality checks are used to eliminate the non-promising designs, that showed biasness with loss of information as an optimality criteria.

\section{Construction Algorithm}

\section{Step 1. Centering of Data}

The initial levels of each factor is centered at zero, i.e. the centre of all factors in the $n$-component zero vector $(0,0, \ldots$, $0)$.

Step 2. Formation of treatment table:

The support points making up the experimental region $\tilde{X}$ are used to form the treatment table.

Step 3. Formation of Latin square plan:

A Latin square plan is formed where the dimension $\mathrm{n} x \mathrm{n}$ is the highest level of the experimental factors.

\section{Step 4. Formation of Groups:}

The groups are formed after superimposing the Latin square plan on the treatment table; where each group is the directrix of complete alphabetical arrangement of the Latin square plan. Thus $k^{\text {th }}$ groups contain (n-2) support points and the resulting groups are $\mathrm{n}$.

Step 5. Construction of $\mathrm{P}$-point trial designs: $\zeta_{p}$.

Support points are assigned to form the $\mathrm{P}$-point designs beginning with those in $\mathrm{g} 1, \mathrm{~g} 2, \ldots$ gn until all $\mathrm{P}$-points have been assigned:

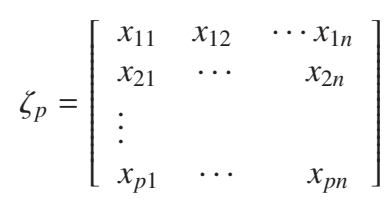

Step 6. Optimality Check:

The design is optimal $\zeta_{p}$ if

(i) $\left\|\sum_{i=1}^{p} x_{i j}^{2}\right\|$ is maximized, $\mathrm{j}=1,2, \ldots \mathrm{p}$. 
(ii) $\left\|\sum_{i=1}^{p} x_{i j}\right\|$ is minimized, $\mathrm{j}=1,2, \ldots \mathrm{p}$.

\section{Illustration and Discussion}

The illustration below shows application of the above construction algorithm on a $3 \times 5$ experimental plan.

The treatment table is as shown in table 2, with the introduction of pseudo factors.

(1) Group formation: The support points are classified into the groups after superimposing the Latin square plan on the treatment table. Each group is gotten from a complete directrix of the alphabetical arrangement of the Latin square plan. Hence;

$$
g_{1}=\left[\begin{array}{cc}
-1 & -2 \\
0 & -1 \\
1 & 0 \\
y & 1 \\
z & 2
\end{array}\right], \quad g_{2}=\left[\begin{array}{cc}
0 & -2 \\
1 & -1 \\
y & 0 \\
z & 1 \\
-1 & 2
\end{array}\right] . \quad g_{3}=\left[\begin{array}{cc}
1 & -2 \\
y & -1 \\
z & 0 \\
-1 & 1 \\
0 & 2
\end{array}\right], \quad g_{4}=\left[\begin{array}{lc}
y & -2 \\
z & -1 \\
-1 & 0 \\
0 & 1 \\
1 & 2
\end{array}\right], \quad g_{5}=\left[\begin{array}{ll}
z & -2 \\
-1 & -1 \\
0 & 0 \\
1 & 1 \\
y & 2
\end{array}\right]
$$

(2) Construction of P-trial point designs, $\zeta_{p}$ : Discard the pseudo factors of each group before making up the P-point design beginning with those in $g_{1}$, then $g_{2}, \cdots, g_{5}$ until P-point have been assigned from the class above, the optimality check is used to eliminate the non-promising ones. Hence the below trial design evolved.

(3)

$$
\zeta_{9 / 13}^{(1)}=\left[\begin{array}{cc}
-1 & -2 \\
0 & -1 \\
1 & 0 \\
-1 & -1 \\
0 & 0 \\
1 & 1 \\
-1 & 0 \\
0 & 1 \\
1 & 2
\end{array}\right] \quad \zeta_{9 / 13}^{(21)}=\left[\begin{array}{ll}
-1 & -2 \\
1 & -1 \\
0 & 1 \\
-1 & -1 \\
-1 & -1 \\
1 & 0 \\
0 & 2 \\
-1 & 0 \\
0 & -2
\end{array}\right]
$$

(4) Application of loss of information optimality checks: With loss of information optimality criterion, the performance of the two promising designs towards optimality show that $\zeta_{9 / 15}^{(1)}$ is optimal since no information is loss on the treatment effects, and this could be seen to show better result as compared to Raghavarao (1971) and Pazman (1981). In Pazman and Raghavarao the construction of P-trial point designs could not be ascertained by the introduction of optimality checks but in our algorithm the optimality checks are used to eliminate non promising designs.

\section{Conclusion}

A technique for the construction of optimal odd fractional design in mixed series experiment is shown to be effective especially with the superimposition of the Latin square, on the experimental treatment table.

Numerical illustration using 3 x 5 experimental plan shows a huge success in its performance.

\section{References}

Banjere, K. S. (1970). Some Observation on the White-Hultquist Procedure for the Construction of Confounding Plans for Mixed Fractional Designs. Annals of Maths. Statist. 41 (1108-1113).

John, P. W. M. (1980). Incomplete Block Designs, Marcel Dekker Inc. Chap. 4, 101-116

Meyer, R. H. (1971). Response Surfaces Methodology, Allyn and Bacon.

Pazman, A. (1987). Foundation of Optimum Experimental Designs, D. Reidel Publishing Company.

Rakfae, B. L. (1999). Combining Elements from Distinct Finite Fields in Mixed Factorials. Ann. Math. Statist. 40, 498-504.

Raghavarao, D. (1971). Construction and Combinatorial Problems in Design of Experiments, J. Wiley. Chap. 8, 441-496

White, D. and Hultquist, R. A. (1965). Construction of Confounding Plans for Mixed Factorial Designs. Ann. Math. Statist. 36, 1256-1271. 


\section{APPENDIX}

Table 1. Treatment table for $3 \times 5$ experiment

\begin{tabular}{|l|l|l|l|l|l|}
\hline & -2 & -1 & 0 & 1 & 2 \\
\hline-1 & $-1,-2$ & $-1,-1$ & $-1,0$ & $-1,1$ & $-1,2$ \\
\hline 0 & $0,-2$ & $0,-1$ & 0,0 & 0,1 & 0,2 \\
\hline 1 & $1,-2$ & $1,-1$ & 1,0 & 1,1 & 1,2 \\
\hline $\mathrm{Y}$ & $\mathrm{Y},-2$ & $\mathrm{Y},-1$ & $\mathrm{Y}, 0$ & $\mathrm{Y}, 1$ & $\mathrm{Y}, 2$ \\
\hline $\mathrm{Z}$ & $\mathrm{Z},-2$ & $\mathrm{Z},-1$ & $\mathrm{Z}, 0$ & $\mathrm{Z}, 1$ & $\mathrm{Z}, 3$ \\
\hline
\end{tabular}

Table 2. 3x5 experiment plan with the introduction of pseudo factors

\begin{tabular}{|c|c|c|c|c|c|c|}
\hline \multicolumn{7}{|l|}{ B } \\
\hline & & -2 & -1 & 0 & 1 & 2 \\
\hline & -1 & $-1-2$ & $-1-1$ & -10 & -11 & -12 \\
\hline \multirow[t]{4}{*}{ A } & 0 & $0-2$ & $0-1$ & 00 & 01 & 02 \\
\hline & 1 & $1-2$ & $1-1$ & 10 & 11 & 12 \\
\hline & $\mathrm{Y}$ & $\mathrm{Y}-2$ & Y -1 & Y 0 & Y 1 & $\mathrm{Y} 2$ \\
\hline & $\mathrm{Z}$ & Z -2 & Z-1 & $\mathrm{ZO}$ & Z 1 & $\mathrm{Z} 2$ \\
\hline
\end{tabular}

